\title{
Association of serum amyloid A protein and peptide fragments with prognosis in renal cancer
}

\author{
SL Wood ', , M Rogers',4, DA Cairns',4, A Paul', D Thompson ${ }^{3}$, NS Vasudev', PJ Selby' and RE Banks*, \\ 'Cancer Research UK Clinical Centre, Leeds Institute of Molecular Medicine, Leeds LS9 7TF, UK; ${ }^{2}$ Department of Urology, St James's University Hospital, \\ Beckett Street, Leeds LS9 7TF, UK; ${ }^{3}$ Department of Clinical Biochemistry and Immunology, Leeds General Infirmary, Great George Street, \\ Leeds LSI 3EX, UK
}

BACKGROUND: In renal cell carcinoma (RCC), the discovery of biomarkers for clinical use is a priority. This study aimed to identify and validate diagnostic and prognostic serum markers using proteomic profiling.

METHODS: Pre-operative sera from 119 patients with clear cell RCC and 69 healthy controls was analysed by surface-enhanced laser desorption/ionisation time-of-flight mass spectrometry with stringent in-house quality control and analysis routines. Following identification of one prognostic peak as a fragment of serum amyloid A (SAA), total serum SAA and CRP were also determined by immunoassay for further validation.

RESULTS: Several peptides were identified as having independent prognostic but not diagnostic significance on multivariable analysis. One was subsequently identified as a I 525 Da fragment of SAA (hazard ratio $(H R)=0.26,95 \% \mathrm{Cl} 0.08-0.85, P=0.026$ ). This was weakly negatively correlated with total SAA, which was also of independent prognostic significance $(\mathrm{HR}=2.46,95 \% \mathrm{Cl} \mid \mathrm{I} .17-5.15$, $P=0.017)$. Both potentially strengthened prognostic models based solely on pre-operative variables.

CONCLUSIONS: This is the first description of the prognostic value of this peptide in RCC and demonstrates proof of principle of the approach. The subsequent examination of SAA protein considerably extends previous studies, being the first study to focus solely on pre-operative samples and describing potential clinical utility in pre-operative prognostic models. British Journal of Cancer (2010) I 03, I0I-1 I I. doi:I0.1038/sj.bjc.6605720 www.bjcancer.com

Published online 8 June 2010

(C) 2010 Cancer Research UK

Keywords: SELDI; proteomics; RCC; prognosis; SAA; CRP

Renal cell carcinoma (RCC) is the most common cancer of the kidney with the main histological subtype being conventional (clear cell), which accounts for around $75 \%$ of cases (Rini et al, 2009) and commonly involves the VHL tumour suppressor gene (Young et al, 2009). Approximately $60 \%$ of patients have clinically localised disease at presentation, with the majority undergoing curative nephrectomy. However, a third of these patients subsequently relapse with metastatic disease. Although several promising markers for RCC have been identified, none currently have been validated and are in routine use (Eichelberg et al, 2009). There is considerable interest in biomarker development generally, both in the context of improving safety and in 'personalised/ individualised' medicine by facilitating earlier and more accurate diagnosis, improving assessment of prognosis, detection of relapse and prediction/monitoring of response (Eichelberg et al, 2009), all of which have additional quality of life and economic benefits. For RCC, diagnostic markers would be particularly valuable in high-risk populations and in patients with unresectable disease, possibly obviating the need for biopsies. Post-surgery, imaging is the key follow-up modality although a multiplicity of regimens have been proposed (Rouviere et al, 2006). Prognostic algorithms

\footnotetext{
*Correspondence: Professor RE Banks;

E-mail: r.banks@leeds.ac.uk

${ }^{4}$ These authors contributed equally to this work.

Received 2 February 2010; revised 4 May 20I0; accepted 5 May 20I0; published online 8 June 2010
}

generally combine TNM staging system with tumour grade, and pathological or clinical factors (Galfano et al, 2008; Lane and Kattan, 2008) but estimates of individual risk are relatively wide and several parameters such as grading are subjective. More recently, the possibility of using algorithms based on pre-operative information has been explored with some success (Cindolo et al, 2003; Raj et al, 2008; Karakiewicz et al, 2009).

Various proteomics-based approaches are being applied to biomarker discovery, each with their own strengths and weaknesses (Issaq et al, 2007; Koomen et al, 2008). One of the approaches that has attracted a lot of interest has been that of surface-enhanced laser desorption/ionisation time-of-flight mass spectrometry (SELDI-TOF-MS) for profiling samples. Significant criticism has been applied to some earlier studies in terms of experimental design, reproducibility and resolution (Kiehntopf et al, 2007; Whelan et al, 2008), but much effort has been made in addressing these issues. We have used SELDI together with stringent in-house quality control (QC) (Cairns et al, 2009) and peak detection methods (Rogers et al, 2003) to investigate whether we can find and validate new serum markers of potential diagnostic or prognostic utility in RCC. On the basis of the subsequent identification of a peak with significant prognostic value as a fragment of serum amyloid A (SAA), we have subsequently analysed total SAA as determined by ELISA and compared the clinical prognostic value of both total and C-terminal fragment of SAA pre-operatively, together with CRP. 


\section{MATERIALS AND METHODS}

\section{Materials}

All routine chemicals were from Sigma-Aldrich (Poole, UK) or VWR Merck (Poole, UK). ProteinChips and calibrants were purchased from Ciphergen Biosystems (now Bio-Rad, Herts, UK) and sinapinic acid from Fluka (Fluka Chemicals Ltd., Buchs, Switzerland). Bio-Suite CM $10 \mu \mathrm{m}$ CXC cation exchange HPLC column $7.5 \times 75 \mathrm{~mm}$ was obtained from Waters (Herts, UK), BondElut $500 \mathrm{mg}$ C18-EWP solid-phase columns from Varian (Varian Inc., Yarnton, Oxford, UK) and C18-zip tips were from Millipore (Herts, UK). Milli-Q water (Millipore (UK) Ltd., Livingston, UK) was used throughout. Monoclonal anti-human SAA (ab18713) was purchased from Abcam (Cambridge, UK) and mouse IgG (MOPC21) from Sigma. Protein-G-coated Dynabeads (cat no: 100-03D) were purchased from Invitrogen (Paisley, UK).

\section{Patients and samples}

Venous blood samples collected from 188 individuals in 2000-2003 (Table 1) comprising 69 healthy controls and 119 patients with clear cell RCC before nephrectomy (102 radical, 6 partial; 11 unknown) or other treatment were analysed. Four

Table I Demographic and clinical details of the patients and healthy controls included in the study

\begin{tabular}{|c|c|}
\hline Characteristic & n (\%) \\
\hline \multicolumn{2}{|l|}{ Gender } \\
\hline Controls - male/female $(n=69)$ & $40: 29(58.0: 42.0)$ \\
\hline RCC - male/female $(n=119)$ & $61: 58(51.3: 48.7)$ \\
\hline \multicolumn{2}{|l|}{ Age at sampling (year) } \\
\hline Controls - median (range) & $62(28,85)$ \\
\hline Mean (s.d.) & $59.0(16.5)$ \\
\hline RCC - median (range) & $63(28,85)$ \\
\hline Mean (s.d.) & $61.1(12.0)$ \\
\hline \multicolumn{2}{|l|}{$p($ Max tumour size; $\mathrm{cm})$} \\
\hline Median (range) & $6(2,16)$ \\
\hline Mean (s.d.) & $6.7(3.4)$ \\
\hline Missing & $5(4.2)$ \\
\hline \multicolumn{2}{|l|}{ ct(Max tumour size; $\mathrm{cm})$} \\
\hline Median (range) & $6(1.7,16)$ \\
\hline Mean (s.d.) & $6.7(3.4)$ \\
\hline Missing & $26(21.9)$ \\
\hline \multicolumn{2}{|l|}{ Fuhrman grade } \\
\hline 1 & $5(7.2)$ \\
\hline 2 & $35(29.4)$ \\
\hline 3 & $43(36.1)$ \\
\hline 4 & $35(29.4)$ \\
\hline Missing & I (0.8) \\
\hline \multicolumn{2}{|l|}{ pT stage } \\
\hline $\mathrm{la}$ & $25(21.0)$ \\
\hline $\mathrm{Ib}$ & $31(26.1)$ \\
\hline 2 & II (9.2) \\
\hline $3 a^{*}$ & $25(21.0)$ \\
\hline $3 b^{*}$ & $25(21.0)$ \\
\hline 4 & I (0.8) \\
\hline Missing & I (0.8) \\
\hline \multicolumn{2}{|l|}{ ctT stage } \\
\hline la & $30(25.2)$ \\
\hline $\mathrm{Ib}$ & $20(16.8)$ \\
\hline 2 & $19(16.0)$ \\
\hline 3 & $21(17.6)$ \\
\hline 4 & $4(3.4)$ \\
\hline Missing & $25(21.0)$ \\
\hline
\end{tabular}

Table I (Continued)

\section{Characteristic}

$N$ stage

0

$\geqslant$

Missing

$M$ stage

0

।

TNM stage

।

॥

$\|$ II***

IV

Missing

MVIIVI

No

Yes

Missing

Necrosis

No

Yes

Missing

Symptoms

Incidental/Asymptomatic

Local

Systemic

Missing

Follow-up (year)

Median (range)

Mean (s.d.)

$2.52(0.01,5.88)$

DFS

Events

Censored

Missing

$49(74.3)$

$5(6.8)$

CSS

Events

Censored

Missing

$14(14.7)$

$76(80.0)$

$5(5.3)$

OS

Events

Censored

$24(25.3)$

$62(65.3)$

Missing

$9(9.5)$

SAA fragment peak intensity Median (range)

Mean (s.d.)

$2.818(0,12.20)$

$3.71(2.31)$

$$
\begin{aligned}
& \text { SAA }\left(m g l^{-1}\right) \\
& \text { Median (range) } \\
& \text { Mean (s.d.) }
\end{aligned}
$$

$6.30(0.80,1470.0)$ $148.10(352.6)$

$\operatorname{CRP}\left(m g l^{-1}\right)$

Median (range)

Mean (s.d.)

$7.15(<5.00,201.6)$ $27.33(45.3)$

Abbreviations: $\mathrm{CRP}=\mathrm{C}$-reactive protein; $\mathrm{CSS}=$ cancer-specific survival; $\mathrm{DFS}=$ disease-free survival; $O S=$ overall survival; $R C C=$ renal cell carcinoma; $S A A=$ serum amyloid A. Includes two and five patients, where $\mathrm{p} T$ = 'at least' $3 \mathrm{a}$ or 3b, respectively. *** Includes three patients for whom a minimum value only of III only could be assigned for stage. For ctT, $3 \mathrm{a}$ and $3 \mathrm{~b}$ are recorded as 3 only, as used in the Karakiewicz et al (2009) model. 
patients subsequently received adjuvant therapy. Samples were obtained after informed consent and with the approval of the local research ethics committee. Blood was collected into Z/serum clot activator tubes (Greiner Bio-One Ltd, Stonehouse, UK) and allowed to clot at room temperature for 30-60 min before being centrifuged at $1800 \mathrm{~g}$ and $20^{\circ} \mathrm{C}$ for $10 \mathrm{~min}$. Serum was aspirated, aliquotted and stored at $-80^{\circ} \mathrm{C}$ until analysis.

This study was designed to follow the STARD and REMARK (Bossuyt et al, 2003; McShane et al, 2005) guidelines for marker identification and evaluation with the primary objective of investigating differential expression in the SELDI profiles of RCC and healthy controls and the planned subset analysis of investigating prognostic significance of the profile. Using pilot data it was shown that in order to detect a two-fold change in peak intensity with a power of $95 \%$ at a significance level of $\alpha=0.001$ (to control false discovery rate) in $90 \%$ of the peaks in the serum profile, 68 samples analysed in duplicate would be required in each group. Additional samples were included in the RCC group as for the prognostic investigation, it was calculated 137 samples would be required at these significance levels and power to detect a hazard ratio (HR) of 5 in a two-fold change in concentration, with a probability of death of $27.5 \%$ in the expected follow-up period. However, as only 119 samples were available, this power is achieved in slightly fewer than $90 \%$ of the peaks.

For the diagnostic study, an initial set of 153 samples (51 normal and 102 RCC) were analysed by SELDI over a period of 5 days. This sample set was then split into a test and training set with similar proportions of cases and controls. A further late test set of 49 samples (26 normal and 23 RCC, including 8 controls and 6 RCC samples previously run in the initial set, to check the longer-term reproducibility) was analysed by SELDI 6 months later. For the prognostic analysis, data from all 119 RCC patients were used.

\section{SELDI profiling}

CM10 chips were prepared and samples applied as previously described (Banks et al, 2005) but using $50 \mathrm{~mm} \mathrm{HEPES/0.1 \%} \mathrm{v/v}$ Triton X-100, pH 7.0 as the wash/dilution buffer. Chips were analysed using a PBS-II SELDI-TOF (Ciphergen Biosystems) with conditions and calibration described in Supplementary methods. All serum samples were analysed in duplicate with each replicate being randomised across days and chips. An experimental QC sample (a serum pool prepared from 20 RCC and 20 normal serum samples) was also included on each chip, assigned to spot A for the first chip, spot $B$ for the second and so on to ensure representative coverage of each spot. Peak detection was carried out using in-house software as previously described (Rogers et al, 2003). All samples and QC spectra were subjected to a range of QC analyses using in-house software (Cairns et al, 2009) and rerun as necessary.

\section{Purification and identification of peak $m / z 1528 \mathrm{Da}$}

In brief (full details in Supplementary Methods), serum diluted with 25 mM HEPES, pH 6.8 was injected onto a BioSuite CM $10 \mu \mathrm{m}$, CXC column. After washing, bound material was eluted with increasing gradient to $1 \mathrm{M} \mathrm{NaCl}$, with the presence of the $1528 \mathrm{Da}$ peak in fractions being monitored by SELDI. Relevant fractions were then concentrated, reduced, alkylated and desalted and applied to a 384-well MALDI plate in a solution of 4-hydroxy- $\alpha$ cyano-cinnamic acid (CHCA) in 50\% aqueous (v/v) acetonitrile/ $0.1 \%(\mathrm{v} / \mathrm{v})$ TFA. Positive-ion MALDI mass spectra were acquired over a mass range of $\mathrm{m} / \mathrm{z} 800-4000$ using a Bruker Ultraflex III (Bruker Daltonics, Bremen, Germany) in reflectron mode with external calibration. Peaks in the relevant mass range were manually selected for MS/MS fragmentation and MS/MS spectra were baseline-subtracted and smoothed; monoisotopic peak detection used a SNAP averaging algorithm with a minimum S/N of 3. Bruker FlexAnalysis software was used to perform the spectral processing and peak list generation. Tandem mass spectral data were submitted to database searching against the NCBInr protein database (2 July 2008) containing 194674 human sequences using Mascot (Matrix Science Ltd., Matrix Science, Boston, MA, USA; version 2.1) with search criteria including: Variable modifications - oxidation (M); Peptide tolerance - 200 p.p.m.; MS/MS tolerance - $0.8 \mathrm{Da}$; and Fixed modification - carbamidomethyl for all alkylated samples.

The identification of the 1525-1528 Da peak was also confirmed by immunoprecipitation of several RCC serum samples. In brief (full details in Supplementary Methods), $20 \mu$ l of serum was mixed with protein G-Dynabeads coupled with either $2 \mu \mathrm{g}$ of mouse antihuman SAA Ab or $2 \mu \mathrm{g}$ of mouse MOPC-21 antibody (negative control). After washing, bound antigens were eluted with $20 \mu \mathrm{l}$ of $100 \mathrm{~mm}$ acetic acid before application to $0.6 \mu \mathrm{l}$ C18-zip-tips. After washing with $0.1 \%(\mathrm{v} / \mathrm{v})$ TFA bound species were eluted onto gold chips using sinappinic acid or CHCA (for mass range $5-20$ or $1-5 \mathrm{kDa}$, respectively). Spectra were acquired using a PCS 4000 Enterprise Protein Chip Reader (Bio-Rad). Mass accuracy was calibrated externally using the all-in-one peptide standard. Peaks were detected after baseline subtraction and spectral calibration.

\section{Immunoassay of SAA and CRP}

Of the original RCC serum samples, 99 were available for subsequent measurement of total SAA at the Supra-Regional Assay service in Sheffield, UK, using rate nephelometry performed on Siemens BNII with a Siemens (Siemens, Deerfield, IL, USA) kit based on a polyclonal antibody. The CRP concentrations were also determined using the Advia 1650 analyser (Bayer, Newbury, UK) in the Clinical Biochemistry Department, St James's University Hospital, Leeds, UK. Normal range for both analytes was $<10 \mathrm{mgl}^{-1}$.

\section{Data analysis}

Diagnosis Peak detected profiles of RCC patients and healthy controls were compared peak-by-peak using a linear mixed effects (LMEs) model similarly to previously reported (Munro et al, 2006) with a random effect for subject, and age and sex included as covariates. The Random Forest classification algorithm (Liaw and Weiner, 2002) selecting 10 random variables at each data split and growing 50000 trees was used to assess the classification ability of the profile, and identified differentially expressed peaks. Receiver operating characteristic (ROC) curve analysis was undertaken to quantify the diagnostic potential of the identified differentially expressed peaks, with the area under the ROC curve (AUC) estimated and a one-sided significance test performed of the null hypothesis that $\mathrm{AUC}>0.5$, against the alternative that $\mathrm{AUC} \leqslant 0.5$ (i.e., no diagnostic ability). When examining multiple significance tests from the LMEs for differential expression (and tests on HRs for prognosis), significance levels of $0.1 \%(P<0.001)$ were used as an ad hoc method of controlling the FDR.

Prognosis Peak detected profiles from RCC patients were averaged across technical replicates and analysed (peak-by-peak) with primary end points, including overall survival (OS), cancerspecific survival (CSS) and disease-free survival (DFS; defined as date of relapse or death from any cause in patients who were disease-free after surgery), timed from the date of nephrectomy. The Kaplan-Meier (KM) method, simple and multivariable Cox proportional hazards regression and the likelihood ratio test (LRT) were used to estimate and evaluate OS, CSS and DFS for the peaks, other known prognostic variables and immunoassay-determined total SAA and CRP. The distribution of the continuous measurements was transformed to the $\log _{2}$ scale to simplify interpretation of estimated HR, and when comparing peak and ELISA measurements the concentrations were scaled by dividing by their standard deviation to make the obtained HRs comparable. The relationship between survival and each standard prognostic clinicopathological 
variable considered for inclusion in the multivariable model (Table 1) was assessed using HRs. In addition, given the interest in using markers pre-operatively to assess prognosis, SAA and CRP were considered together with the variables of age, gender, symptom score, CT-derived size, $\mathrm{T}$ stage and presence or absence of metastatic disease, as used in the pre-operative predictive model of Karakiewicz et al (2009) to assess their independent pre-operative predictive value. The assumption of proportional hazards in the Cox regression was checked using the test of Grambsch and Therneau for simple and multivariable analysis.

Analysis was undertaken using the R Environment for Statistical Computing ( $\mathrm{R}$ Development Core Team, Vienna) applying functions in the nlme and survival libraries and in Stata 9.0 (College Station, TX, USA) using the lroc function.

\section{RESULTS}

\section{Diagnosis}

Over all spectra, a total of 383 different peaks were detected with a median number of 92 in the healthy controls and 95 in the RCC spectra. Three peaks $(\mathrm{m} / \mathrm{z} 4802.1,6675.9,7341.1 \mathrm{Da})$ were significantly differently expressed between cases and controls. Although these peaks were highly significant in terms of differences in means, the distributions of the peak intensities overlapped (Supplementary Figure 1). The intra-class correlation coefficients are also quite small indicating only moderate reproducibility (Supplementary Table 1). The limited value of these peaks as diagnostic biomarkers was confirmed by ROC curve analysis, in which each peak was found to have only limited predictive ability with $\mathrm{AUC} \approx 60 \%$. The ability of the profile to classify cases and controls was assessed using the Random Forest. Examining the training set, first test set and blind test set resulted in a high out-of-bag classification error (44\%) and poor classification in terms of sensitivity/specificity, that is in the training set $73 \% / 22 \%$, in the test set $72 \%, 27 \%$ and in the blind validation set $76 \% / 31 \%$, respectively. Considering that these results were achieved in a comparison of healthy controls and RCC patients, a subsequent comparison including benign disease controls in which even poorer performance could be anticipated was not performed.

\section{Prognosis}

Details of the length of follow-up and number of events (CSS, DFS and OS) are given in Table 1 . CSS rates $(95 \% \mathrm{CI})$ from nephrectomy for all RCC patients were $87.5 \%(80.8-94.7 \%)$ for 1 year and $71.3 \%$ $(61.4-83.1 \%)$ for 3 years. DFS rates $(95 \% \mathrm{CI})$ from nephrectomy for all RCC patients were $91.0 \%(84.4-98.1 \%)$ for 1 year and $77.3 \%$ $(66.7-89.5 \%)$ for 3 years. Overall survival rates from nephrectomy for all RCC patients were $86.5 \%$ (79.7-94.0\%) for 1 year and $66.5 \%$ $(56.2-78.8 \%)$ for 3 years. Median CSS, DFS and OS were not reached in this follow-up period. The characteristics of the cohort of RCC patients were checked according to known prognostic factors (Table 2) and, as expected, significant associations were identified between CSS and several clinicopathological features including grade, stage, microvascular invasion and necrosis.

Considering all RCC patients, no peaks were identified that were significantly associated with DFS $(P<0.001)$, but 12 peaks were identified that were significantly associated with CSS $(P<0.001-$ Supplementary Table 2). Six of these peaks (three of which are shown in Figure 1) have independent prognostic value $(P<0.001)$ when fitting a multivariable model with age, sex, $\mathrm{pT}, \mathrm{M}$ and maximum tumour size included as covariates. One peak was subsequently identified, namely, the peak of approximate $\mathrm{m} / \mathrm{z}$ $1525-1528 \mathrm{Da}$, the intensity of which was inversely related to survival, as the $\mathrm{C}$ terminal 13 amino acid fragment of SAA (P02735) with a predicted mass of $1525.8 \mathrm{Da}$ and Mascot score of 41, indicating significant homology and common to both SAA-1 and SAA-2 (Figure 2). The estimated HR for this peak in the univariate analysis is shown in Table 2. It is encouraging that the HR in the multivariable analysis $(\mathrm{HR}=0.26,95 \% \mathrm{CI} 0.08-0.85, P=0.026)$ is similar to that in the single variable analysis and the $95 \%$ CIs overlap to an extent also. As can be seen from examining the HR, $P$ columns in Table 2, no prognostic variables provided any evidence of violating the proportional hazards assumptions in both the simple and multivariable models. The identity of this peak was also confirmed by immunoprecipitation (Figure 3) with several additional peaks being specifically precipitated including the $11 \mathrm{kDa}$ cluster of three peaks previously identified (Tolson et al, 2004; Engwegen et al, 2007) as intact SAA (11.68 kDa), des-R SAA $(11.5 \mathrm{kDa})$ and des-RS SAA (11.4 kDa). Peaks at 9.29 and $7.61 \mathrm{kDa}$, which may correspond to previously observed proteolytic breakdown products of SAA by cathepsin-B action (Rocken et al, 2005), were also seen (data not shown).

The summary statistics for SAA as determined by immunoassay are shown in Table 2, and show weak evidence of a negative correlation of $r=-0.16$ (95\% CI -0.35 to $0.04, P=0.11)$ with the intensity of the $1528 \mathrm{Da}$ SAA fragment. SAA was also strongly positively correlated with CRP $(r=0.88,95 \%$ CI $0.82-0.92$, $P<10^{-15}$ ) and showed evidence of an association (details in Supplementary Results) with grade $\left.P<10^{-5}\right)$, pT $(P=0.002)$, CT-determined T-stage $\left(P<10^{-3}\right)$, TNM stage $(P=0.0003)$, $\mathrm{N}$ stage $\left(P<10^{-3}\right)$ and symptoms score $(P=0.007)$. In addition, there was evidence of a positive correlation with tumour size determined by CT (Pearson $r=0.416, P<10^{-4}$ ) or pathology $\left(r=0.368, P<10^{-3}\right)$. However, there was no evidence of an association with $\mathrm{M}$ stage $(P=0.1611)$. There was no evidence of significant association or correlation between these clinicopathological variables and the SAA peak fragment.

Figure 4 shows CSS for the SAA fragment of $1528 \mathrm{Da}$, mature SAA and CRP with increases in mature SAA and CRP being associated with poorer survival. It is clear that the relationship between hazard of death and concentration of SAA as measured by immunoassay is in an opposing direction to the SAA fragment (Figure 4). This is confirmed by examining the HR for SAA for univariate analysis in Table $2\left(\mathrm{HR}=3.77,95 \% \mathrm{CI} 2.35-6.05, P<10^{-7}\right)$. In fact, it is almost exactly the reciprocal of the $\mathrm{HR}$ for the peak at $1528 \mathrm{Da}$ $\left(\mathrm{e}^{(-\log (3.77))}=0.27\right)$, a near perfect inverse relationship. The survival function for CRP is very similar to that for SAA, which is possibly not surprising considering their strong positive correlation.

The results of the multivariable analysis to test for independent prognostic ability are shown in Table 2 . The estimated HRs in the preoperative predictive model of Karakiewicz et al (2009) were contained in the $95 \%$ CIs for HR for all, except one of the variables in Table 4 (local vs asymptomatic symptoms) indicating the transferability of this model to our data. In addition, SAA is shown to have independent prognostic ability (LRT, $P=0.017$ ). The $\mathrm{HR}$ of the multivariable analysis for SAA was similar, but reduced to that for the univariate analysis, indicating that some of the relationship with survival time explained by SAA is explained by the other variables in the model. The SAA fragment was also an independent prognostic factor but when CRP was added to the model, SAA or the SAA fragment were no longer independent prognostic factors (SAA; LRT, $P=0.208$ ), but adding CRP to the model containing SAA and the other pre-operative factors was independently prognostic (LRT, $P=0.004$ ).

This analysis was similarly undertaken for OS and DFS (Supplementary Tables 3 and 4). The SAA fragment and immunoassay SAA measurement were found to be significantly related with OS $(P<0.05)$ on single variable analysis, and on multivariable analysis there was some evidence of independent prognostic ability for the SAA immunoassay measurement (LRT, $P=0.080$ ). However, these measurements were not found to be significantly independently prognostic of DFS (LRT, $P>0.05$ ), although the HRs were similar to those found in CSS/OS. The CRP was shown to be highly significantly related to OS $\left(P<10^{-7}\right)$ and 
Table 2 Univariate and multivariable prognostic modelling results for all RCC patients for CSS using Cox proportional hazards regression

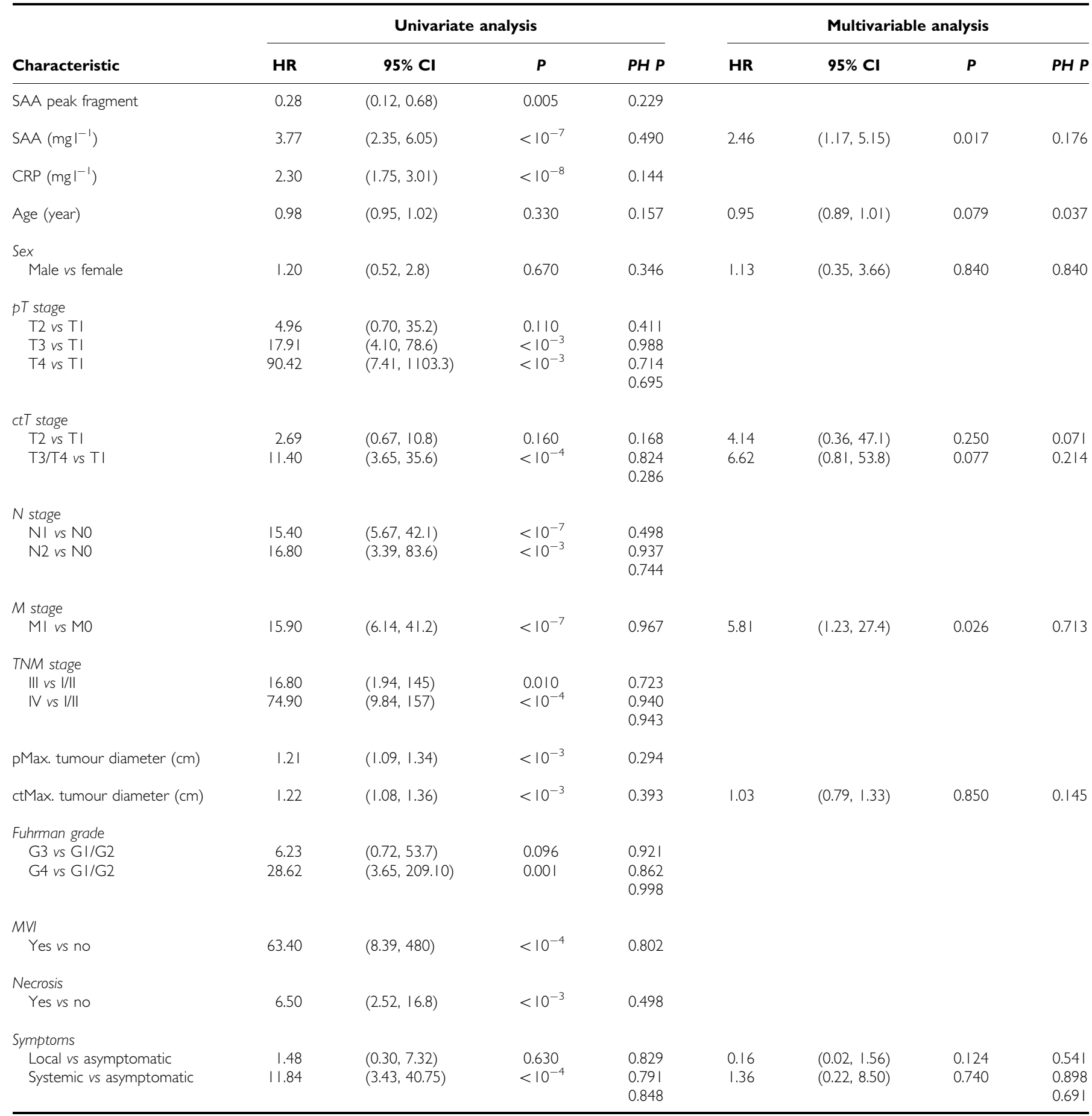

Abbreviations: $\mathrm{Cl}=$ confidence interval; $\mathrm{CRP}=\mathrm{C}$-reactive protein; $\mathrm{CSS}=$ cancer-specific survival; $\mathrm{HR}=$ hazard ratio; $\mathrm{RCC}=$ renal cell carcinoma; $\mathrm{SAA}=$ serum amyloid $\mathrm{A}$. Multivariable results refer to the model considering immunoassay-determined SAA. PH P columns refer to the test for evidence of violation of the proportional hazards assumption.

DFS $\left(P<10^{-3}\right)$ in RCC. Similar analysis was undertaken for CSS, DFS and OS considering non-cancer deaths, and no relationship was found between the SAA fragment or total SAA or CRP $(P>0.05)$, indicating the specific nature of the relationship of these factors with prognosis and RCC.

\section{DISCUSSION}

This study demonstrates an important proof of principle of the ability of a proteomic profiling approach to successfully detect a potential prognostic serum biomarker, specifically a C-terminal fragment of SAA, for renal cancer. Few such examples that exist in the cancer literature currently and mainly address diagnosis. The validity of the identified C-terminal fragment of SAA in serum as being of independent prognostic significance in RCC with higher levels being associated with better survival was further demonstrated by its inverse correlation with intact SAA, as measured by immunoassay and, importantly, almost perfectly inverse HRs between the two. Critically, both this fragment and intact SAA have been shown for the first time to provide additional information 


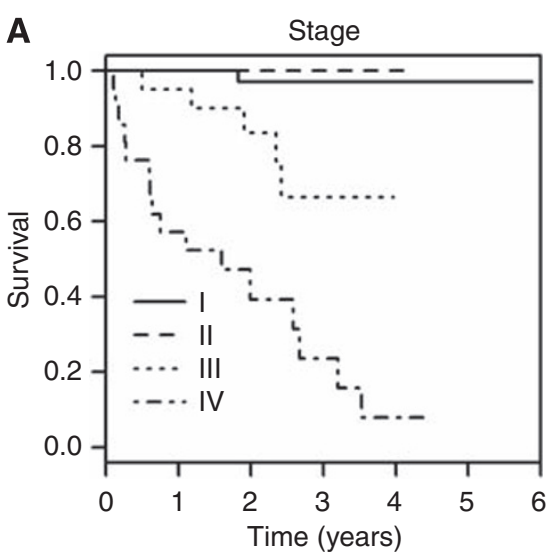

B
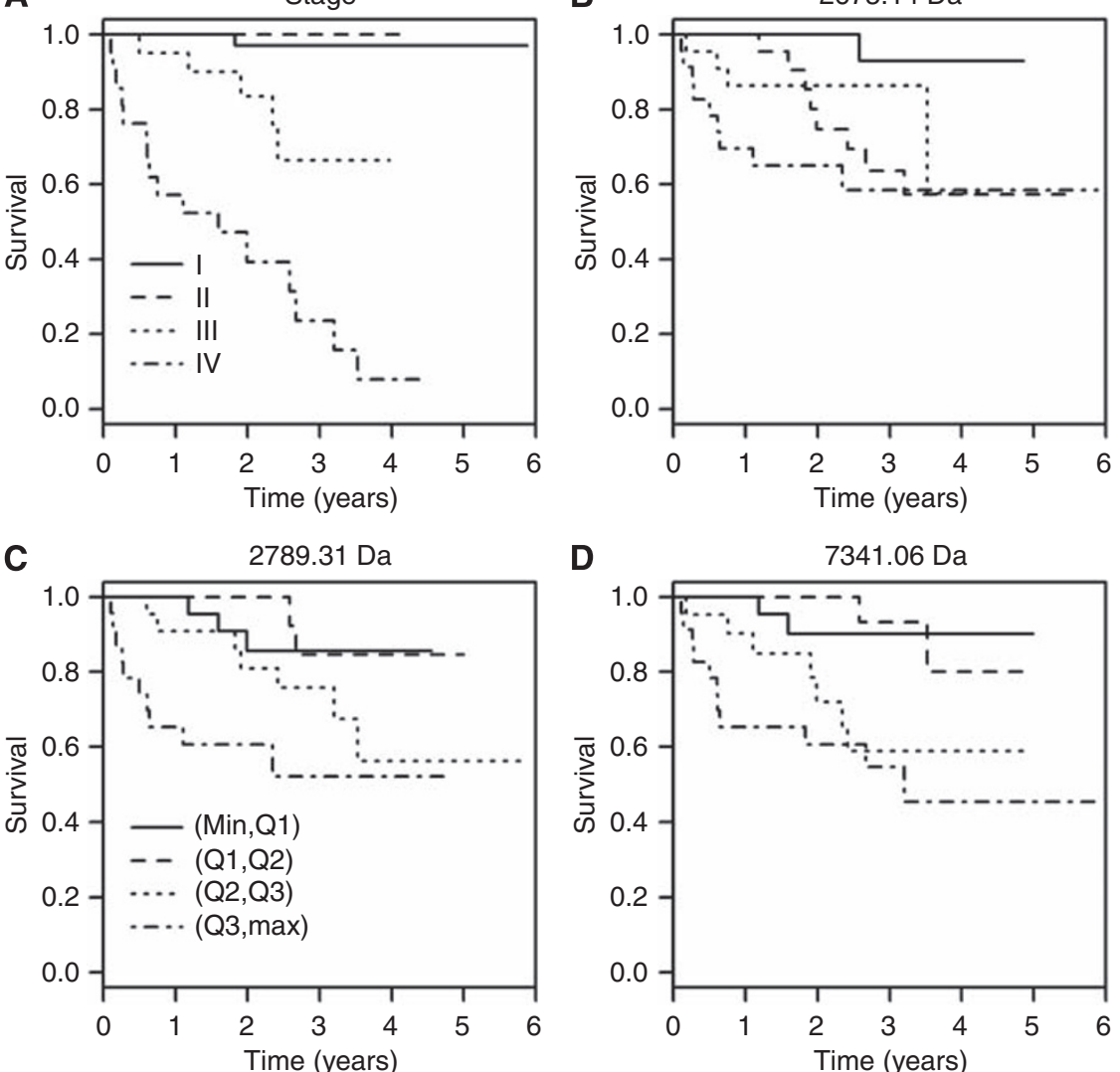

Figure I Example of three peaks from SELDI profile that have independent prognostic ability, as determined by multivariable analysis using Cox proportional hazards regression when correcting for age, sex, pT, M and pathology-determined tumour size. Panels show KM estimated survival function for peak intensity split into quartiles with TNM stage shown for comparison in $(\mathbf{A})$. The legend for the quartiles in $(\mathbf{C})$ applies to $(\mathbf{B}$ and $\mathbf{D})$.

when used in combination with other clinical variables in preoperative prognostication.

There has been increasing interest in SAA, a major apolipoprotein of HDL, in cancer with the recognition that it is not just an acute phase protein synthesised by the liver but is also produced extrahepatically including by many cancers (Malle et al, 2009). This includes renal cancer, particularly aggressive disease, on the basis of microarray gene expression profiling studies and/or quantitative RT-PCR (Liou et al., 2004; Kosari et al., 2005; Paret et al, 2010). Epithelial cell expression of SAA-1 was subsequently demonstrated by immunohistochemistry of clear cell RCC samples as part of a TMA, correlating with a poor clinical outcome, although only 15 of 87 cases were positive (Paret et al, 2010). As such, it may therefore be both a tumour-derived factor in addition to being a systemic reflection of the cytokines produced either by the tumour or as a result of the host response and is present in elevated concentrations in serum in many types of cancer (Malle et al, 2009). Sustained chronic elevation of SAA, such as that observed in some inflammatory diseases and cancers, including renal cancer is thought to contribute to amyloidosis.

The ability of MALDI or SELDI-based fluid profiling to deliver new potential biomarkers is still largely unproven (Findeisen and Neumaier, 2009). The critical nature of sample handling (Banks et al, 2005), the relatively poor mass resolution of some platforms and lack of QC procedures have been problematic (Whelan et al, 2008; Findeisen and Neumaier, 2009). Although discrimination between groups on the basis of profiles may be achieved, the failure to demonstrate long-term robustness or to easily identify peaks of interest has limited progress (Whelan et al, 2008). This study has adopted stringent QC and analysis methods together with consistent sample processing and has shown results of potential utility, although again only one peak was able to be identified despite extensive efforts. With MALDI-TOF-MS, the peak was observed at an $m / z 1525.81 \mathrm{Da}$ (1525-1528 Da by SELDITOF-MS) compared with a theoretical mass of $1525.73 \mathrm{Da}$. The theoretical pI is 9.01, which would be expected to bind to the chip surface under the conditions used in this study, unlike SAA minus the $C$ terminal 13 amino acids with a pI of 6.57. Similarly, intact SAA and the N-terminal truncated des-R and des-RS forms of SAA-1 in RCC serum as previously described (Tolson et al, 2004; Engwegen et al, 2007) were not detected by profiling as expected, given their predicted pIs of 5.5-6.5 but were present in RCC serum as revealed by our immunoprecipitation-MS experiments, along with other fragments of SAA.

Functions of SAA relating to tumourigenesis (Malle et al, 2009) include binding to extracellular matrix components with subsequent potential modification of cell binding, enhancement of plasminogen activation and stimulation of matrix metalloproteinase (MMP) production, reported in several cell types including renal cancer cell lines (Paret et al, 2010). Matrix metalloproteinases can cleave SAA proteins (Stix et al, 2001), and C-terminal cleavage of SAA by cathepsin-B (Rocken et al, 2005) and cathepsin-D has been described (van der Hilst et al, 2009). There is no defined motif for a specific protease cleavage site in SAA, which would generate the $1528 \mathrm{Da}$ fragment described here. Numerous peptides present in plasma or serum are largely generated from proteins such as fibrinogen, thrombin and complement components (Richter et al, 1999; Koomen et al, 2005; Zhou et al, 2006; Peng et al, 2009). However, 'disease-specific' N- and C-terminal exoprotease activities superimposed on these coagulation and complement-based proteolytic events have been proposed to generate disease-specific serum peptide signatures, including for 
A

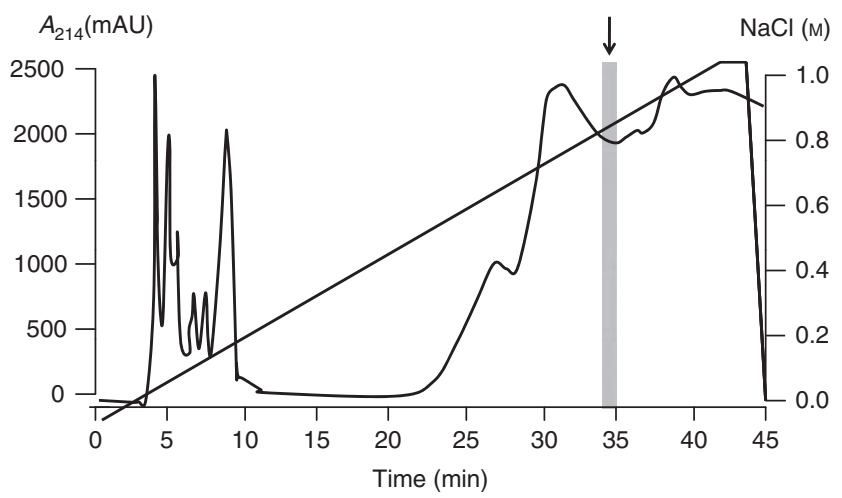

C

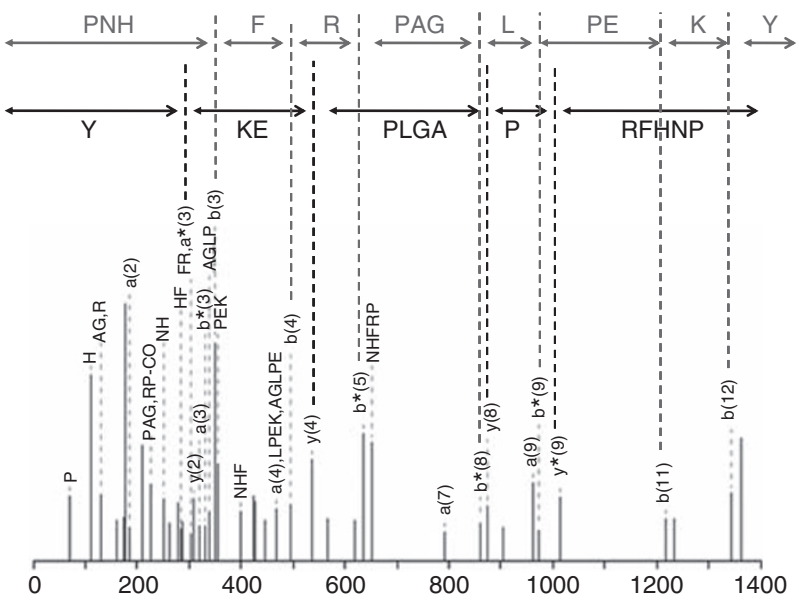

B

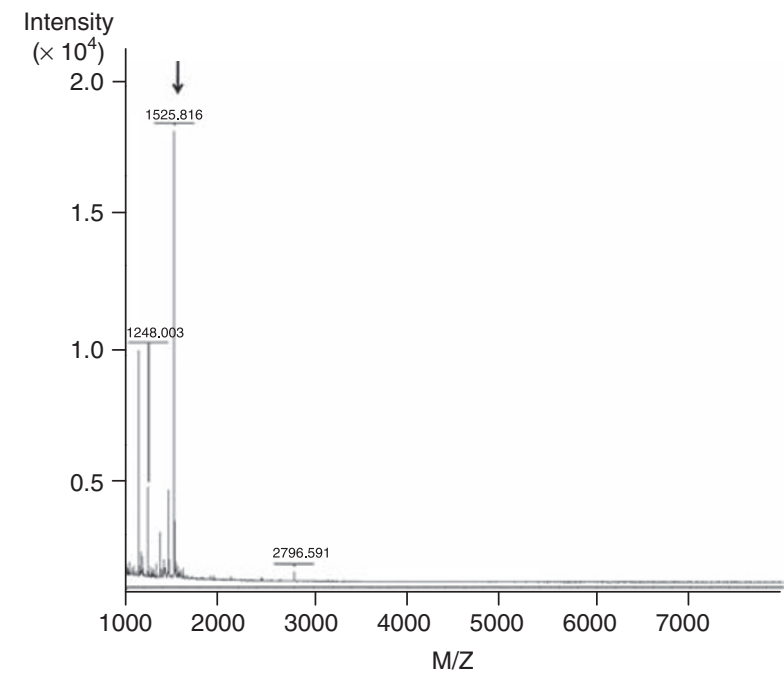

D
MKLLTGLVFC SLVLGVSSRS FFSFLGFEAFD GARDMWRAYS

41

81

\section{DMREANYIGS DKYFHARGNY DAAKRGPGGV WAAEAISDAR}

Figure 2 Identification of the peak at 1526-1528 Da as an N-terminal fragment of SAA. (A) Elution profile during chromatography of donor serum showing the elution of the peak (shaded box) from a CMIO column as monitored by SELDI, (B) MS spectrum of fraction with an arrow indicating the precursor ion, (C) MSMS spectrum for the sequenced peptide, (D) primary amino-acid sequence of serum amyloid A protein with the sequenced fragment illustrated in bold.

prostate, bladder and breast cancer (Villanueva et al, 2006). Such a mechanism may account for the SAA fragment identified here. Several SELDI profiling studies have detected elevated concentrations of $11.7 \mathrm{kDa}$ mature form and peak clusters at $11.4-11.7 \mathrm{kDa}$ lacking one or two amino acids in various cancers, including renal cancer and particularly metastatic disease (Tolson et al, 2004; Engwegen et al, 2007; Malle et al, 2009), with some subsequent validation through immunoassay of SAA and demonstration of high prognostic significance, for example, in melanoma (Findeisen et al, 2009). These and other novel $\mathrm{N}$-terminal truncated forms and/or post-translationally modified forms of SAA1 and 2 have been described in plasma samples from healthy controls and patients with inflammatory diseases (Ducret et al, 1996; Kiernan et al, 2003), although their significance is as yet unknown.

The possibility of serum SAA as measured by immunoassay being of prognostic value in RCC has previously been examined in two studies. The first involved 72 patients with RCC (47 clear cell), where poor CSS was associated with higher SAA and SAA was an independent prognostic factor on multivariate analysis, although few parameters were examined (Kimura et al, 2001). A more recent study involving 98 RCC patients (70 clear cell) found that SAA concentration correlated significantly with $\mathrm{T}$ stage and grade. Again only CSS was examined and SAA was found to be independently significantly associated with survival, although only $\mathrm{T}$ and $\mathrm{M}$ stage and grade were examined. (Ramankulov et al, 2008). Interestingly, significantly higher concentrations of SAA were found in patients with metastatic disease with the AUC for a ROC analysis of metastatic $v s$ non-metastatic disease of 0.86 . However, our study did not confirm this, with no evidence of metastatic diagnostic potential being seen. The earlier study was slightly complicated by the fact that almost half of the patients with metastatic disease were sampled 1-76 months post-nephrectomy when metastases were diagnosed, whereas in our study all samples were obtained before any treatment. To what extent these assays detect SAA fragments is not known but is very likely to be assayspecific and dependent on antibody combinations and epitopes recognised.

Our study clearly adds considerably to the literature being based solely on patients with conventional clear cell RCC and all samples being pre-treatment. In addition, OS, CSS and DFS were all examined and with multiple variables for the models including specifically a focus on pre-operative ones. The strong correlation between CRP and SAA is to be expected, given their commonality in terms of being induced by similar cytokines, although kinetics in the acute situation differ with SAA being much more sensitive and rapid in terms of increase and subsequent decline 

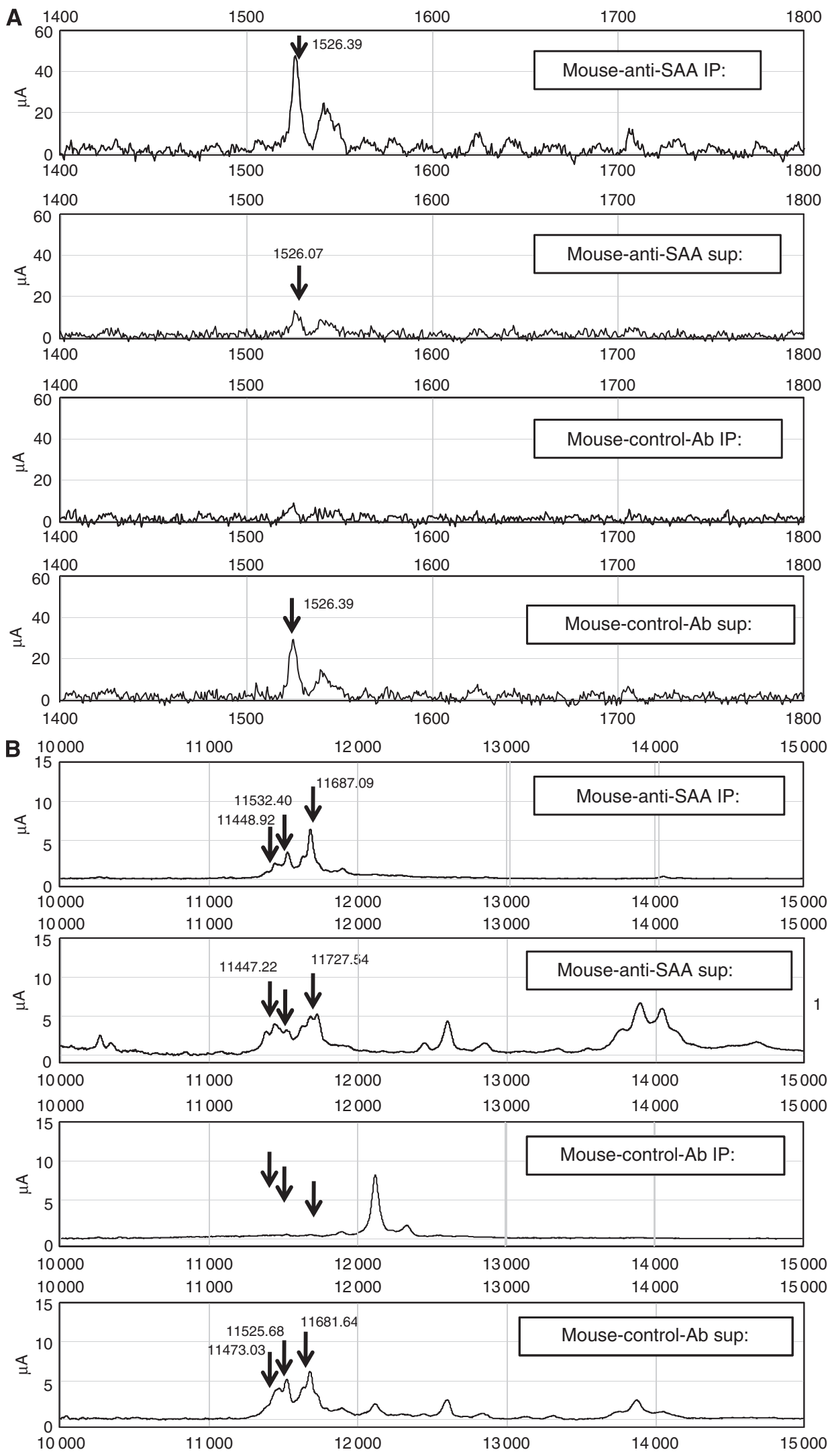

Figure 3 Immunoprecipitation of SAA (comparison of mouse anti-human SAA with control isotype-matched mouse $\lg G$ ) from RCC sera analysed by SELDI-TOF MS. (A) Region of the spectra showing the $1528 \mathrm{Da}$ SAA fragment. (B) Region of the spectra showing intact SAA with the three arrows indicating the positions of the des-RS SAA-I ( I I.4 kDa), des-R SAA- I ( I I.5 kDa) and SAA- I ( I .68 kDa) isoforms previously described (Tolson et al, 2004; Engwegen et al, 2007). 
A

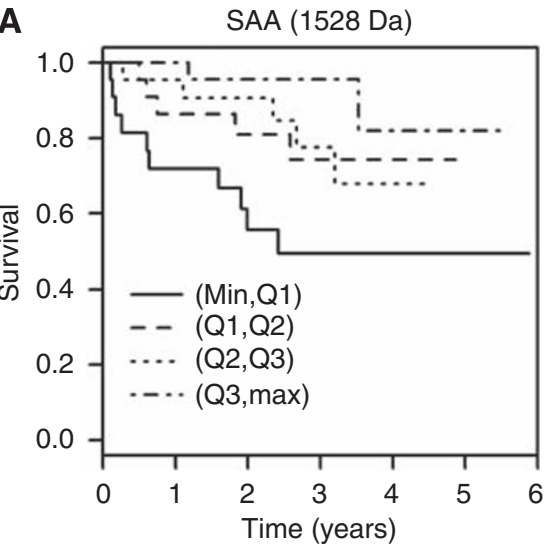

B

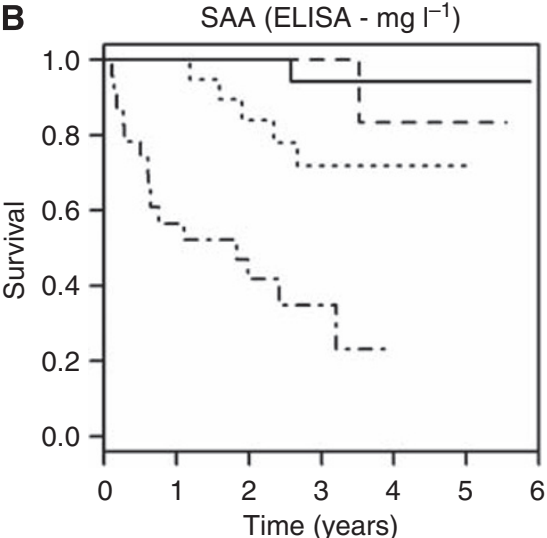

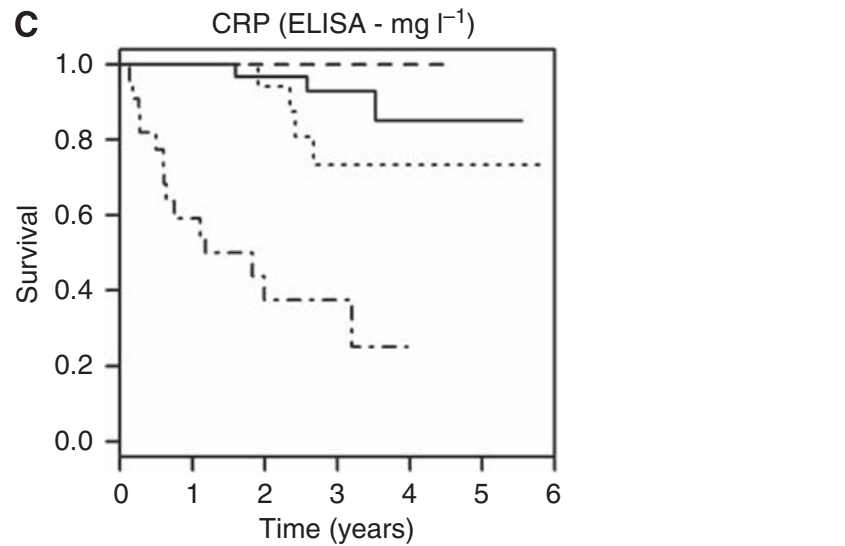

Figure 4 Plots of KM estimated survival functions for (A) peak intensity of SAA fragment (I528.9 Da), (B) SAA concentration as determined by immunoassay and, (C) CRP concentration, in each case split into quartiles. The legend in $\mathbf{A}$ applies to all panels but with the values for minimum, QI, Q2, Q3 and maximum being specific to each.

(Banks et al, 1995; Malle and De Beer, 1996). Both CRP and SAA were very highly independently statistically significant for prognosis pre-operatively, although SAA lost this independence when CRP was added, implying that CRP may provide additional information. There is a body of evidence supporting CRP as an independent prognostic marker in RCC (Ito et al, 2006; Karakiewicz et al, 2007; Komai et al, 2007; Ramsey et al, 2008; Iimura et al, 2009) and this may arise as a result of production by the tumour cells themselves (Jabs et al, 2005). Clearly, although correlated with many clinical variables, including $\mathrm{T}$ stage, nodal status, grade, TNM stage and tumour size, both SAA and CRP provide additional information of interest.

These findings warrant further study in larger patient groups prospectively to determine conclusively the prognostic value of SAA and CRP either alone or in combination with each other and other prognostic variables available pre-operatively. Possible utility in longitudinal monitoring should also be examined with specificity being determined by repeated measurements to check for transient rises due to episodes of acute inflammatory illnesses. Clearly, measurement of SAA by immunoassay is superior to the SAA fragment in terms of prognostic ability power, and therefore

\section{REFERENCES}

Banks RE, Forbes MA, Storr M, Higginson J, Thompson D, Raynes J, Illingworth JM, Perren TJ, Selby PJ, Whicher JT (1995) The acute phase protein response in patients receiving subcutaneous IL-6. Clin Exp Immunol 102: 217-223 developing an assay specifically for this fragment would not be justified at present given the challenges involved, although it could potentially provide additional information if a disease-specific cleavage. Robust assays for SAA and CRP are already in place in hospital clinical chemistry laboratories, providing relatively inexpensive and objective measurement and facilitating future studies.

\section{ACKNOWLEDGEMENTS}

The financial support of Cancer Research UK is gratefully acknowledged. We are also grateful to the patients for consenting to participate, Annie Stanley for technical assistance, Jenny Barrett for statistical discussions, Adam Dowd and Jerry Thomas of the Technology facility at the University of York for protein sequencing and the staff of the Urology, Pathology and Oncology departments at St James's University Hospital in Leeds.

Supplementary Information accompanies the paper on British Journal of Cancer website (http://www.nature.com/bjc) 
Bossuyt PM, Reitsma JB, Bruns DE, Gatsonis CA, Glasziou PP, Irwig LM, Lijmer JG, Moher D, Rennie D, de Vet HC (2003) Towards complete and accurate reporting of studies of diagnostic accuracy: the STARD initiative. Standards for Reporting of Diagnostic Accuracy. Clin Chem 49(1): $1-6$

Cairns DA, Perkins DN, Stanley AJ, Thompson D, Barrett JH, Selby PJ, Banks RE (2009) Integrated multi-level quality control for proteomic profiling studies using mass spectrometry. BMC Bioinformatics 9: 519

Cindolo L, De La Taille A, Messina G, Romis L, Abbou CC, Altieri V, Rodriguez A, Patard JJ (2003) A preoperative clinical prognostic model for non-metastatic renal cell carcinoma. BJU Int 92: 901-905

Ducret A, Bruun CF, Bures EJ, Marhaug G, Husby G, Aebersold R (1996) Characterization of human serum amyloid A protein isoforms separated by two-dimensional electrophoresis by liquid chromatography/ electrospray ionization tandem mass spectrometry. Electrophoresis 17: 866-876

Eichelberg C, Junker K, Ljungberg B, Moch H (2009) Diagnostic and prognostic molecular markers for renal cell carcinoma: a critical appraisal of the current state of research and clinical applicability. Eur Urol 55: 851-863

Engwegen JY, Mehra N, Haanen JB, Bonfrer JM, Schellens JH, Voest EE, Beijnen JH (2007) Validation of SELDI-TOF MS serum protein profiles for renal cell carcinoma in new populations. Lab Invest 87(2): $161-172$

Findeisen P, Neumaier M (2009) Mass spectrometry-based proteomics profiling as diagnostic tool in oncology: current status and future perspective. Clin Chem Lab Med 47: 666-684

Findeisen P, Zapatka M, Peccerella T, Matzk H, Neumaier M, Schadendorf D, Ugurel S (2009) Serum amyloid A as a prognostic marker in melanoma identified by proteomic profiling. J Clin Oncol 13: 2199-2208

Galfano A, Novara G, Iafrate M, Cavalleri S, Martignoni G, Gardiman M, D'Elia C, Patard JJ, Artibani W, Ficarra V (2008) Mathematical models for prognostic prediction in patients with renal cell carcinoma. Urol Int 80: $113-123$

Iimura Y, Saito K, Fujii Y, Kumagai J, Kawakami S, Komai Y, Yonese J, Fukui I, Kihara K (2009) Development and external validation of a new outcome prediction model for patients with clear cell renal cell carcinoma treated with nephrectomy based on pre-operative serum C-reactive protein and TNM classification: the TNM-C score. J Urol 181: $1004-1012$

Issaq HJ, Xiao Z, Veenstra TD (2007) Serum and plasma proteomics. Chem Rev 107: $3601-3620$

Ito K, Asano T, Yoshii H, Satoh A, Sumitomo M, Hayakawa M (2006) Impact of thrombocytosis and C-reactive protein elevation on the prognosis for patients with renal cell carcinoma. Int J Urol 13: 1365 - 1370

Jabs WJ, Busse M, Kruger S, Jocham D, Steinhoff J, Doehn C (2005) Expression of C-reactive protein by renal cell carcinomas and unaffected surrounding renal tissue. Kidney Int 68: 2103-2110

Karakiewicz PI, Hutterer GC, Trinh QD, Jeldres C, Perrotte P, Gallina A, Tostain J, Patard JJ (2007) C-reactive protein is an informative predictor of renal cell carcinoma-specific mortality: a European study of 313 patients. Cancer 110: 1241-1247

Karakiewicz PI, Suardi N, Capitanio U, Jeldres C, Ficarra V, Cindolo L, De La Taille A, Tostain J, Mulders PFA, Bensalah K, Artibani W, Salomon L, Zigeuner R, Valeri A, Descotes J-L, Rambeaud J-J, Mejean A, Montorsi F, Bertini R, Patard J-J (2009) A preoperative prognostic model for patients treated with nephrectomy for renal cell carcinoma. Eur Urol 55: $287-295$

Kiehntopf M, Siegmund R, Deufel T (2007) Use of SELDI-TOF mass spectrometry for identification of new biomarkers: potetential and limitations. Clin Chem Lab Med 45: 1435-1449

Kiernan UA, Tubbs KA, Nedelkov D, Niederkofler EE, Nelson RW (2003) Detection of novel truncated forms of human serum amyloid A protein in human plasma. FEBS Lett 537: 166-170

Kimura M, Tomita Y, Imai T, Saito T, Katagiri A, Ohara-Mikami Y, Matsudo T, Takahashi K (2001) Significance of serum amyloid a on the prognosis in patients with renal cell carcinoma. Cancer 92(8): $2072-2075$

Komai Y, Saito K, Sakai K, Morimoto S (2007) Increased preoperative serum C-reactive protein level predicts a poor prognosis in patients with localized renal cell carcinoma. BJU Int 99: 77-80

Koomen JM, Haura EB, Bepler G, Sutphen R, Remily-Wood ER, Benson K, Hussein M, Hazlehurst LA, Yeatman TJ, Hildreth LT, Sellers TA, Jacobsen PB, Fenstermacher DA, Dalton WS (2008) Proteomic contributions to personalized cancer care. Mol Cell Proteomics 7: 1780- 1794
Koomen JM, Li D, Xiao LC, Liu TC, Coombes KR, Abbruzzese J, Kobayashi R (2005) Direct tandem mass spectrometry reveals limitations in protein profiling experiments for plasma biomarker discovery. J Proteome Res 4(3): 972-981

Kosari F, Parker AS, Kube DM, Lohse CM, Leibovich BC, Blute ML, Cheville JC, Vasmatzis G (2005) Clear cell renal carcinoma: gene expression analyses identify a potential signature for tumour aggressiveness. Clin Cancer Res 11(14): $5128-5139$

Lane BR, Kattan MW (2008) Prognostic models and algorithms in renal cell carcinoma. Urol Clin North Am 35: 613-625

Liaw A, Weiner M (2002) Classification and regression by randomForest. Rnews 2: $18-22$

Liou LS, Shi T, Duan ZH, Sadhukhan P, Der SD, Novick AA, Hissong J, Skacel M, Almasan A, DiDonato JA (2004) Microarray gene expression profiling and analysis in renal cell carcinoma. BMC Urol 4: 9

Malle E, De Beer FC (1996) Human serum amyloid A (SAA) protein: a prominent acute-phase reactant for clinical practice. Eur J Clin Invest 26: $427-435$

Malle E, Sodin-Semrl S, Kovacevic A (2009) Serum amyloid A: an acute phase protein involved in tumour pathogenesis. Cell Mol Life Sci 66: $9-26$

McShane LM, Altman DG, Sauerbrei W, Taube SE, Gion M, Clark GM (2005) Reporting recommendations for tumor marker prognostic studies (REMARK). J Natl Cancer Inst 97(16): 1180-1184

Munro NP, Cairns DA, Clarke P, Rogers M, Stanley AJ, Barrett JH, Harnden P, Thompson D, Eardley I, Banks RE, Knowles MA (2006) Urinary biomarker profiling in transitional cell carcinoma. Int J Cancer 119: $2642-2650$

Paret C, Schön Z, Szponar A, Kovacs G (2010) Inflammatory protein serum amyloid A1 marks a subset of conventional renal cell carcinomas with fatal outcome. Eur Urol 859-866

Peng J, Stanley AJ, Cairns D, Selby PJ, Banks RE (2009) Using the protein chip interface with quadrupole time-of-flight mass spectrometry to directly identify peaks in SELDI profiles - initial evaluation using low molecular weight serum peaks. Proteomics 9: 492-498

Raj GV, Thompson RH, Leibovich BC, Blute ML, Russo P, Kattan MW (2008) Preoperative nomogram predicting 12-year probability of metastatic renal cancer. J Urol 179: 2146-2151

Ramankulov A, Lein M, Johannsen M, Schrader M, Miller K, Loening SA, Jung K (2008) Serum amyloid A as indicator of distant metastases but not as early tumor marker in patients with renal cell carcinoma. Cancer Lett 269: 85-92

Ramsey S, Lamb GWA, Aitchison M, McMillan DC (2008) Prospective study of the relationship between the systemic inflammatory response, prognostic scoring systems and relapse-free and cancer-specific survival in patients undergoing potentially curative resection for renal cancer. Br J Urol 101: 959-963

Richter R, Schulz-Knappe P, Schrader M, Standker L, Jurgens M, Tammen H, Forssmann WG (1999) Composition of the peptide fraction in human blood plasma: database of circulating human peptides. J Chromatogr $B$ Biomed Sci Appl 726(1-2): 25-35

Rini BI, Campbell SC, Escudier B (2009) Renal cell carcinoma. Lancet 373: $1119-1132$

Rocken C, Menard R, Buhling F, Vockler S, Raynes J, Stix B, Kruger S, Roessner A, Kahne T (2005) Proteolysis of serum amyloid A and AA amyloid proteins by cystine proteases: cathepsin B generates AA amyloid proteins and cathepsin L may prevent their formation. Ann Rheum Dis 64: $808-815$

Rogers MA, Clarke P, Noble J, Munro NP, Paul A, Selby PJ, Banks RE (2003) Proteomic profiling of urinary proteins in renal cancer by surface enhanced laser desorption ionisation (SELDI) and neural network analysis: identification of key issues affecting potential clinical utility. Cancer Res 63(20): 6971-6983

Rouviere O, Bouvier R, Negrier S, Badet L, Lyonnet D (2006) Nonmetastatic renal-cell carcinoma: is it really possible to define rational guidelines for post-treatment follow-up? Nat Clin Pract Oncol 3(4): 200-213

Stix B, Kahne T, Sletten K, Raynes J, Roessner A, Rocken C (2001) Proteolysis of AA amyloid fibril proteins by matrix metalloproteinases-1, -2, and -3. Am J Pathol 159: 561-570

Tolson J, Bogumil R, Brunst E, Beck H, Elsner R, Humeny A, Kratzin H, Deeg M, Kuczyk M, Mueller GA, Mueller CA, Flad T (2004) Serum protein profiling by SELDI mass spectrometry: detection of multiple variants of serum amyloid alpha in renal cancer patients. Lab Invest 84(7): $845-856$ 


\section{$\underset{\mathrm{OPEN}}{\mathrm{BJC}} \mathrm{O}$}

van der Hilst JCH, Kluve-Beckerman B, van der Meer JW, Simon A (2009) Cathepsin D activity protects against development of type AA amyloid fibrils. Eur J Clin Invest 39: 412-416

Villanueva J, Shaffer DR, Philip J, Chaparro CA, Erdjument-Bromage H, Olshen AB, Fleisher M, Lilja H, Brogi E, Boyd J, Sanchez-Carbayo M, Holland EC, Cordon-Cardo C, Scher HI, Tempst P (2006) Differential exoprotease activities confer tumor-specific serum peptidome patterns. J Clin Invest 116(1): 271-284

Whelan LC, Power KAR, McDowell DT, Kennedy J, Gallagher WM (2008) Applications of SELDI-MS technology in oncology. J Cell Mol Med 12: 1535-1547

Young AC, Craven RA, Cohen D, Taylor C, Booth C, Harnden P, Cairns DA, Astuti D, Gregory W, Maher ER, Knowles MA, Joyce A, Selby PJ, Banks RE (2009) Analysis of VHL gene alterations and their relationship to
SAA and prognosis in renal cancer

SL Wood et al

clinical parameters in sporadic conventional renal cell carcinoma. Clin Cancer Res 15: $7582-7592$

Zhou M, Prieto DA, Lucas DA, Chan KC, Issaq HJ, Veenstra TD, Conrads TP (2006) Identification of the SELDI ProteinChip human serum retentate by microcapillary liquid chromatography-tandem mass spectrometry. J Proteome Res 5: 2207-2216

(c) (1) (2) This work is licensed under the Creative Commons BY NC SA Attribution-NonCommercial-Share Alike 3.0 Unported License. To view a copy of this license, visit http://creativecommons. org/licenses/by-nc-sa/3.0/ 